Disclosure of Interests: None declared

DOI: 10.1136/annrheumdis-2019-eular.8237

\section{FRI0571 MEASUREMENT PERFORMANCE OF REDUCED VERSIONS OF MUSCLE STRENGTH TOOLS IN JUVENILE DERMATOMYOSITIS}

Silvia Rosina ${ }^{1}$, Giulia Camilla Varnier ${ }^{2}$, Angela Pistorio ${ }^{1}$, Alessandro Consolaro ${ }^{1}$, Clarissa Pilkington ${ }^{3}$, Susan Maillard ${ }^{3}$, Adele Civino ${ }^{4}$, Elena Tsitsami ${ }^{5}$, Jaime de Inocencio ${ }^{6}$, Marija Jelusic ${ }^{7}$, Jelena Vojinovic ${ }^{8}$, Graciela Espada ${ }^{9}$, Balahan Makay ${ }^{10}$, Maria Katsikas ${ }^{11}$, Polixeni Pratsidou-Gertsi ${ }^{12}$ Dragana Lazarevic ${ }^{13}$, Anand Prahalad Rao ${ }^{14}$, Nicolino Ruperto ${ }^{1}$, Angelo Ravelli ${ }^{1}$ ${ }^{1}$ IRCCS Istituto Giannina Gaslini, Genova, Italy; ${ }^{2}$ Università degli Studi di Genova, Genova, Italy; ${ }^{3}$ Great Ormond Street Hospital, London, United Kingdom; ${ }^{4}$ Ospedale Vito Fazzi, Lecce, Italy, ${ }^{5}$ Children's Hospital Agia Sofia, Athens, Greece; ${ }^{6}$ Hospital Universitario 12 de Octubre, Madrid, Spain; ${ }^{7}$ University Hospital Center Zagreb, Zagreb, Croatia; ${ }^{8}$ University of Nis, Nis, Serbia; ${ }^{9} \mathrm{Hospital}$ de Ninos Ricardo Gutierez, Buenos Aires, Argentina; ${ }^{10}$ Dokuz Eylul University Hospital, Izmir, Turkey; ${ }^{11}$ Hospital de Pediatria Juan P. Garrahan, Buenos Aires, Argentina; ${ }^{12}$ Hippokration General Hospital, Thessaloniki, Greece; ${ }^{13}$ Clinic Center, Nis, Serbia; ${ }^{14}$ Manipal Hospital, Bangalore, India

Background: Assessment of muscle strength is a fundamental component of the clinical evaluation of children with juvenile dermatomyositis (JDM) Regular measurement of muscle strength in daily care requires the availability of simple and quick muscle assessment tools.

Objectives: To investigate whether reduced versions of the MMT8 and CMAS are equally reliable as the original tools.

Methods: The following 4 reduced instruments were devised: 1) MMT4 (score 0-40), including 4 items of MMT8 (neck flexors, deltoid middle, gluteus maximus, and gluteus medius); 2) MMT6 (score 0-60), composed of the same items of MMT4 plus biceps brachii and quadriceps; 3) head lift time of CMAS (0-120 seconds or 0-5 points); 4) sum of CMAS head lift time in points and the 6 sit-ups maneuvers of CMAS (score 0-11). Validation was conducted according to OMERACT filter on 213 patients followed in standard clinical care at 13 international pediatric rheumatology centers and evaluated at baseline and after a median of 5.9 months.

Results: All reduced instruments revealed strong correlations $(r>0.7)$ with muscle activity VAS and total DAS, moderate correlations $(r=0.4-$ 0.7 ) with physician's global VAS, muscle DAS, skin activity VAS, pain VAS, parent's overall wellbeing VAS, and CK. Correlations with skin DAS and fatigue VAS were low $(r<0.4)$. Cronbach's alpha was excellent (0.92-0.95) for all reduced tools for which this property could be assessed. SRM was good-to-moderate $(0.60-0.91)$ for all reduced instruments in patients judged as improved by the physician. All reduced tools discriminated strongly between patients classified in different disease activity states by the physician $(p<0.0001)$, and between patients whose parents were satisfied or not satisfied with their children's disease status $(p<0.0001)$. Overall, the metrologic performance of the reduced instruments was comparable to that of MMT8 and CMAS.

Conclusion: We found that reduced versions of the MMT8 and CMAS have good metrologic properties and perform similarly to the original tools in a population of patients followed in standard clinical care. Our results suggest that these simplified and shortened instruments could serve as surrogate for the complete measures in routine practice, particularly in a busy clinical setting.

\section{REFERENCES:}

[1] Measures of adult and juvenile dermatomyositis, polimyositis, and inclusion body myositis. Rider LG, Werth VP, Huber AM, Alexanderson H, Rao AP, et al. Arthritis Care Res (Hoboken) 2011;63:S118-57.

[2] Clinical assessment in juvenile dermatomyositis. Ravelli A, Ruperto N, Trail L, Felici E, Sala E, et al. Autoimmunity 2006;39:197-203.

Disclosure of Interests: Silvia Rosina: None declared, Giulia Camilla Varnier: None declared, Angela Pistorio: None declared, Alessandro Consolaro Grant/research support from: AbbVie, Pfizer, Clarissa Pilkington: None declared, Susan Maillard: None declared, Adele Civino: None declared, Elena Tsitsami: None declared, Jaime de Inocencio: None declared, Marija Jelusic: None declared, Jelena Vojinovic: None declared, Graciela Espada: None declared, Balahan Makay Speakers bureau: Enzyvant, Novartis, Roche, Abbvie, Maria Katsikas: None declared, Polixeni Pratsidou-Gertsi: None declared, Dragana Lazarevic: None declared, Anand Prahalad Rao: None declared, Nicolino Ruperto Grant/research support from: The Gaslini Hospital, where NR works as full-time public employee, has received contributions (> 10.000 USD each) from the following industries in the last 3 years: BMS, Eli-Lilly, GlaxoSmithKline, F Hoffmann-La Roche, Janssen, Novartis, Pfizer, Sobi. This funding has been reinvested for the research activities of the hospital in a fully independent manner, without any commitment with third parties., Consultant for: Received honoraria for consultancies or speaker bureaus $<10.000$ USD each) from the following pharmaceutical companies in the past 3 years: Ablynx, AbbVie, Astrazeneca-Medimmune, Biogen, Boehringer, Bristol-Myers Squibb, Eli-Lilly, EMD Serono, GlaxoSmithKline, Hoffmann-La Roche, Janssen, Merck, Novartis, Pfizer, R-Pharma, SanofiServier, Sinergie, Sobi and Takeda., Speakers bureau: Received honoraria for consultancies or speaker bureaus (< 10.000 USD each) from the following pharmaceutical companies in the past 3 years: Ablynx, AbbVie, Astrazeneca-Medimmune, Biogen, Boehringer, Bristol-Myers Squibb, Eli-Lilly, EMD Serono, GlaxoS mithKline, Hoffmann-La Roche, Janssen, Merck, Novartis, Pfizer, RPharma, SanofiServier, Sinergie, Sobi and Takeda., Angelo Ravelli Grant/ research support from: Angelini, AbbVie, Bristol-Myers Squibb, Johnson \& Johnson, Novartis, Pfizer, Reckitt Benkiser, and Roche, Consultant for: Angelini, AbbVie, Bristol-Myers Squibb, Johnson \& Johnson, Novartis, Pfizer, Reckitt Benkiser, and Roche, Speakers bureau: Angelini, AbbVie, Bristol-Myers Squibb, Johnson \& Johnson, Novartis, Pfizer, Reckitt Benk iser, and Roche

DOI: 10.1136/annrheumdis-2019-eular.2228

\section{FRI0572 DISABILITY AND HEALTH-RELATED QUALITY OF LIFE OUTCOMES IN PATIENTS WITH SYSTEMIC JUVENILE IDIOPATHIC ARTHRITIS TREATED WITH TOCILIZUMAB IN A PHASE 3 RANDOMIZED CONTROLLED TRIAL}

Nicolino Ruperto ${ }^{1}$, Chen Chen ${ }^{2}$, Alberto Martini ${ }^{1}$, Graciela Espada ${ }^{1}$, Rik Joos ${ }^{1}$ Jonathan Akikusa ${ }^{1}$, Jeffrey Chaitow ${ }^{1}$, Maria Luz Gámir Gámir ${ }^{1}$, Yukiko Kimura ${ }^{2}$, Christoph Rietschel' ${ }^{1}$, Daniel Siri ${ }^{1}$, Elzbieta Smolewska ${ }^{1}$, Heinrike Schmeling ${ }^{2}$, Diane Brown ${ }^{2}$, Fabrizio De Benedetti ${ }^{1}$, Daniel J. Lovell ${ }^{2}$, Bin Huang ${ }^{2}$, Hermine Brunner ${ }^{2} .{ }^{1}$ Paediatric Rheumatology International Trials Organisation (PRINTO), Genoa, Italy, ${ }^{2}$ Pediatric Rheumatology Collaborative Study Group (PRCSG), Cincinnati Children's Hospital Medical Center, Cincinnati, United States of America

Background: Tocilizumab (TCZ) intravenous (IV) formulation was approved for the treatment of patients with systemic juvenile idiopathic arthritis (sJIA) based on the results of a large phase 3 clinical trial. Physical function, measured by the Childhood Health Assessment QuestionnaireDisability Index (CHAQ-DI), and health-related quality of life (HRQOL), measured by the Child Health Questionnaire (CHQ), were evaluated. Objectives: To examine measures of disability and HRQOL in patients with SJIA treated with TCZ IV for up to 2 years in post hoc analysis of data from the phase 3 trial of TCZ.

Methods: Changes within 3 months of treatment initiation with TCZ (baseline) were compared between TCZ- and placebo (PBO)-treated patients using CHAQ-DI, pain global assessment (Pain-GA), physician global assessment, and patient global assessment (Pt-GA) using analysis of variance adjusted for treatment group. Changes in CHAQ-DI overall and domain scores and changes in $\mathrm{CHQ}$ domain and summary scores from baseline to 2 years were compared for patients treated with TCZ using the unpaired $t$ test.

Results: Patients with sJIA experienced clinically relevant improvement in physical function (CHAQ-DI) and reduction of pain (Pain-GA). Mean (SD) CHAQ-DI scores for patients treated with PBO and TCZ were $1.7(0.8)$ for both groups at baseline and $1.2(1.0)$ and $0.9(0.8)$, respectively, at week 12 (week 12 mean difference, -0.2 ; median [range] change from baseline to week 12 difference, $-0.3[-0.6$ to 0.0$])$. These patients also had significantly improved $\mathrm{CHQ}$ socialization, behavior, mental health, and psychosocial summary scores after 3 months compared with those receiving PBO (Figure 1). Improvement in all CHAQ-DI domains over 2 years was observed with TCZ treatment (Figure 2); improvement rates in patient well-being (Pt-GA) were $87.7 \%$. Similar improvements in physical function were observed in patients with polyarticular JIA in the phase 3 trial that led to approval of TCZ IV in these patients (not shown). There was also significant improvement $(p<0.05)$ in most domains of HRQOL ( $\mathrm{CHQ}$ domain scores) in patients with SJIA; of note, patients experienced marked improvement in mean scores from baseline to week 104 for pain/discomfort (31.7 to 75.3 ), self-esteem (61.0 to 76.0), mental health (62.1 to 76.8 ), and social limitation-emotional (52.6 to 86.2 ).

Conclusion: Two years of TCZ treatment resulted in statistically significant and clinically relevant improvements in function and $\mathrm{HRQOL}$ in patients with SJIA. 

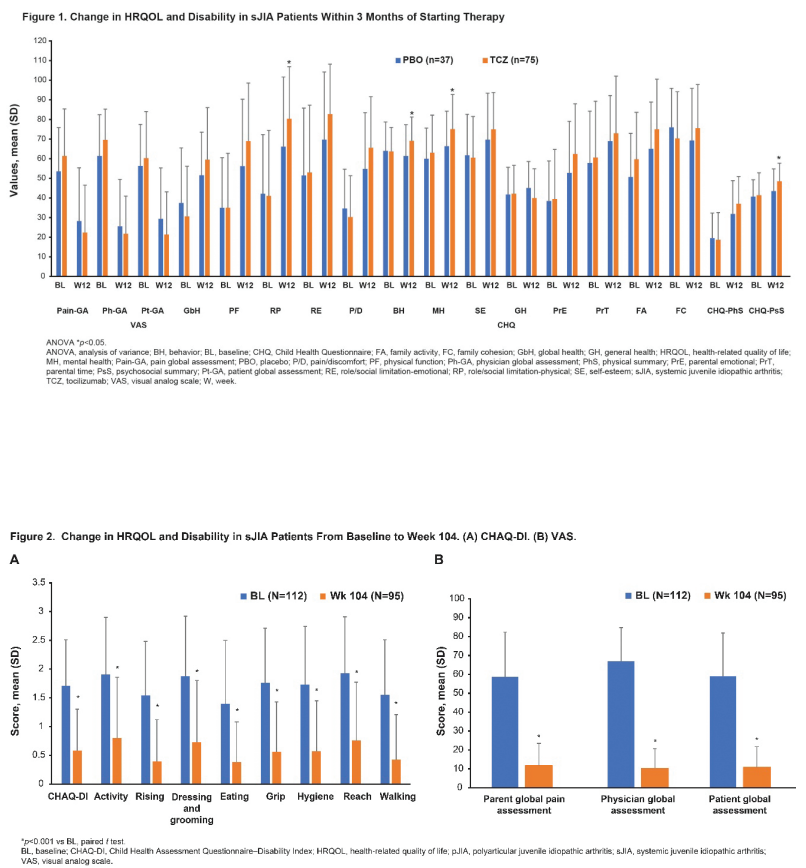

Disclosure of Interests: Nicolino Ruperto Grant/research support from: The Gaslini Hospital, where NR works as full-time public employee, has received contributions (> 10.000 USD each) from the following industries in the last 3 years: BMS, Eli-Lilly, GlaxoSmithKline, F Hoffmann-La Roche, Janssen, Novartis, Pfizer, Sobi. This funding has been reinvested for the research activities of the hospital in a fully independent manner, without any commitment with third parties., Consultant for: Received honoraria for consultancies or speaker bureaus (< 10.000 USD each) from the following pharmaceutical companies in the past 3 years: Ablynx, AbbVie, Astrazeneca-Medimmune, Biogen, Boehringer, Bristol-Myers Squibb, Eli-Lilly, EMD Serono, GlaxoSmithKline, Hoffmann-La Roche, Janssen, Merck, Novartis, Pfizer, R-Pharma, SanofiServier, Sinergie, Sobi and Takeda., Speakers bureau: Received honoraria for consultancies or speaker bureaus ( $<10.000$ USD each) from the following pharmaceutical companies in the past 3 years: Ablynx, AbbVie, Astrazeneca-Medimmune, Biogen, Boehringer, Bristol-Myers Squibb, Eli-Lilly, EMD Serono, GlaxoSmithKline, Hoffmann-La Roche, Janssen, Merck, Novartis, Pfizer, RPharma, SanofiServier, Sinergie, Sobi and Takeda., Chen Chen: None declared, Alberto Martini: None declared, Graciela Espada: None declared, Rik Joos: None declared, Jonathan Akikusa: None declared, Jeffrey Chaitow: None declared, Maria Luz Gámir Gámir: None declared, Yukiko Kimura Consultant for: Novartis, SOBI, Christoph Rietschel: None declared, Daniel Siri: None declared, Elzbieta Smolewska: None declared, Heinrike Schmeling Grant/research support from: F. Hoffmann-La Roche Ltd, Diane Brown: None declared, Fabrizio De Benedetti Grant/research support from: Abbvie, SOBI, Novimmune, Roche, Novartis, Sanofi, Pfizer, Daniel J Lovell Consultant for: Consulting fees and/or honoraria from Astra Zeneca, Wyeth Pharma, Amgen, Abbott, Pfizer, F. Hoffmann-La Roche, Novartis, UBC, Takeda, GSK, Boehringer, and Celgene, Bin Huang: None declared, Hermine Brunner Grant/research support from: Bristol-Myers Squibb, Pfizer, Consultant for: Pfizer, Bristol-Myers Squibb, Janssen, Novartis, Lilly, Roche, GlaxoSmithKline, Sanofi, Speakers bureau: Novartis, Roche

DOI: 10.1136/annrheumdis-2019-eular.3392

\section{FRI0573 \\ COGNITIVE IMPAIRMENT IN CHILDHOOD-ONSET SYSTEMIC LUPUS ERYTHEMATOSUS: EARLY DETECTION WITH MR SPECTROSCOPY AND ITS ASSOCIATION WITH MOG ANTIBODIES}

Huseyin Kilic ${ }^{1}$, Sezgin Sahin ${ }^{2}$, Mekiya Filiz Toprak ${ }^{3}$, Gokce Hale Hatay ${ }^{4}$, Kubra Yilmaz ${ }^{1}$, Amra Adrovic ${ }^{2}$, Kenan Barut ${ }^{2}$, Esin Ozturk Isik ${ }^{4}$, Erdem Tuzun ${ }^{5}$, Osman Kizilkilic ${ }^{6}$, Sema Saltik ${ }^{7}$, Ozgur Kasapcopur ${ }^{2}$. ${ }^{1}$ Istanbul UniversityCerrahpasa, Cerrahpasa Medical School, Pediatric Neurology, Istanbul, Turkey; ${ }^{2}$ Istanbul University-Cerrahpasa, Cerrahpasa Medical School, Pediatric Rheumatology, İstanbul, Turkey, ${ }^{3}$ Bezmialem Vakif University, School of Medicine, Pediatric Neurology, istanbul, Turkey; ${ }^{4}$ Institute of Biomedical Engineering, Bogazici University, Istanbul, Turkey; ${ }^{5}$ Istanbul University, Aziz Sancar Institute of Experimental Medicine, Neuroscience, Istanbul, Turkey; ${ }^{6}$ Istanbul UniversityCerrahpasa, Cerrahpasa Medical School, Neuroradiology, ístanbul, Turkey;

${ }^{7}$ Istanbul University-Cerrahpasa, Cerrahpasa Medical School, Pediatric Neurology, istanbul, Turkey

Background: Systemic lupus erythematosus (SLE) is a chronic inflammatory disease characterized by the presence of various autoantibodies. Unnoticed and progressive cognitive impairment may develop in the course of disease even without overt neuropsychiatric (NP) features. Some authors attributed this mild impairment to the immune-mediated myelinopathy. Evidence exists that myelin oligodendrocyte glycoprotein (MOG) might act as a mediator of interactions between myelin and the immune system.

Objectives: To detect the role of MOG-Ab in neurologic manifestations of childhood-onset SLE (cSLE) and to better delineate the actual grade of cognitive dysfunction by neurocognitive tests in patients without overt NP features (non-NPSLE). Moreover, we aimed to identify the localization of pathology at molecular level by analyzing the presence of any correlation between various metabolite peaks in MR spectroscopy (MRS) and the severity of cognitive dysfunction in non-NPSLE children.

Methods: MOG-Ab levels were studied in all healthy subjects $(n=28)$ and in all patients with (NPSLE =9) and without (non-NPSLE=36) overt neuropsychiatric manifestations. All of the non-NPSLE group and healthy group underwent brain MR and MRS examination. However, only 20 subjects in each group met the MRS imaging standards for evaluation. In the nonNPSLE group, 29 cSLE patients were further evaluated by neurocognitive tests. Sixteen children with non-NPSLE were assessed by both MRS and neurocognitive tests.

Results: The mean age of the SLE patients at study time was 16.22 3.22 years. MOG-Ab was detected positive neither in CSLE nor in healthy group.

In children with non-NPSLE, verbal IQ ranged from 40 to 108 (mean: $79.06 \pm 17.66$ ), performance IQ ranged from 42 to 111 (mean: 92.03 \pm 16.28 ), and full-scale IQ ranged from 40 to 106 (mean: 84.31 \pm 16.39 ). There were 15 patients $(51 \%)$ in non-NPSLE group with a full-scale IQ under 85. There was no significant difference between the non-NPSLE group and healthy subjects in terms of choline, $\mathrm{N}$-aspartic acid (NAA), creatine, NAA/creatine and choline/creatine.

A negative correlation was observed between the NAA/Creatine level of the left frontal white matter in MRS and the stroop test time (measures the ability to shift a perceptual set with changing demands which is a function of the frontal lobes) $(p=0.015 ; r=0.596)$.

Conclusion: More than a half of our patients in non-NPSLE group were found to have a full-scale IQ under 85. Cognitive impairment may develop insidiously in CSLE children even without any overt symptom or sign. There was no association of MOG-Ab with CSLE, whether neuropsychiatric manifestations present or not. A causal relationship between immune-mediated myelinopathy and neuropsychiatric involvement/cognitive impairment could not be suggested, since there has been no patient with positive MOG-Ab and there has been no difference in choline, cholin/creatine between CSLE patients and healthy subjects. Decrease in the NAA Creatine level of the left frontal white matter in MRS, which is a finding of neuronal loss, may be used as a first sign of cognitive impairment in patients with CSLE.

Disclosure of Interests: : None declared

DOI: 10.1136/annrheumdis-2019-eular.7658 